\title{
Effects of xanthine oxidase inhibitors on renal function and blood pressure in hypertensive patients with hyperuricemia
}

\begin{abstract}
Kentaro Kohagura ${ }^{1,2}$, Takeshi Tana ${ }^{3}$, Akira Higa ${ }^{3}$, Masanobu Yamazato ${ }^{2}$, Akio Ishida ${ }^{2}$, Kazufumi Nagahama², Atsushi Sakima ${ }^{2}$, Kunitoshi Iseki ${ }^{4,5}$ and Yusuke Ohya ${ }^{1,2}$

Hyperuricemia may promote the progression of hypertension and renal dysfunction. However, the effects of hyperuricemia treatment on blood pressure and renal function in adult hypertensive patients with hyperuricemia remain unclear. A total of 137 hypertensive patients with hyperuricemia (96 men and 41 women; mean age of 67 years) who recently started taking xanthine oxidase inhibitors (allopurinol or febuxostat) as outpatients were recruited. Serum uric acid level, estimated glomerular filtration rate (eGFR, $\mathrm{ml} \mathrm{min}^{-1}$ per $1.73 \mathrm{~m}^{2}$ ) and blood pressure $(\mathrm{mm} \mathrm{Hg}$ ) were retrospectively compared immediately before and shortly after starting treatment with xanthine oxidase inhibitors. The mean blood pressure and the eGFR immediately before starting treatment were $128 / 71 \mathrm{~mm} \mathrm{Hg}$ and $44.6 \mathrm{ml} \mathrm{min}^{-1}$ per $1.73 \mathrm{~m}^{2}$, respectively. Although the eGFR decreased from 46.6 to $44.6 \mathrm{ml} \mathrm{min}^{-1}$ per $1.73 \mathrm{~m}^{2}$ before starting treatment with xanthine oxidase inhibitors, it increased to $46.2 \mathrm{ml} \mathrm{min}^{-1}$ per $1.73 \mathrm{~m}^{2}(P=0.001$, compared with immediately before treatment) without any significant changes in blood pressure after the administration of xanthine oxidase inhibitors. Multiple regression analysis revealed that the increase in eGFR after starting xanthine oxidase inhibitor treatment positively correlated with the changes in systolic blood pressure and negatively correlated with the changes in uric acid levels and the use of renin-angiotensin system inhibitors. These results suggest that xanthine oxidase inhibitors may delay the progression of renal dysfunction in adult hypertensive patients with hyperuricemia. Hypertension Research (2016) 39, 593-597; doi:10.1038/hr.2016.37; published online 14 April 2016
\end{abstract}

Keywords: chronic kidney disease; uric acid; xanthine oxidase inhibitor

\section{INTRODUCTION}

Many epidemiologic studies have suggested that hyperuricemia may have a pathogenic role in the development of hypertension ${ }^{1-3}$ and the progression of kidney disease. ${ }^{4-6}$ In accordance with this hypothesis, urate-lowering drugs have been reported to reduce blood pressure in adolescents with essential hypertension ${ }^{7}$ who are generally characterized by a short duration of hypertension, a lack of metabolic abnormality and scarce organ damage such as renal dysfunction. However, limited information is available regarding the effects of urate-lowering drugs on blood pressure in adult patients. In contrast to children, adults with hypertension often present with other comorbidities, such as diabetes mellitus and dyslipidemia, ${ }^{8}$ and are already undergoing treatment with antihypertensive agents. In hypertensive patients with diabetes mellitus, a newly developed elevation of uric acid has been associated with an increased risk for chronic kidney disease (CKD) progression. ${ }^{9}$ Previous interventional studies suggest that xanthine oxidase inhibitors may delay the progression of renal dysfunction in patients with renal insufficiency. ${ }^{10,11}$ However, the effects of xanthine oxidase inhibitors on blood pressure and renal function in adult hypertensive patients with hyperuricemia are not clear.

Thus, we conducted a historical cohort study among the hypertensive patients with hyperuricemia who had recently started treatment with xanthine oxidase inhibitors, including allopurinol and febuxostat, in our outpatient clinic of internal medicine.

\section{METHODS}

Settings and participants

A total of 485 patients with hyperuricemia undergoing treatment with allopurinol or febuxostat at the University of the Ryukyus Hospital and the Shuri-Jokamachi Clinic, Okinawa, Japan, between 1 October 2013 and 30 September 2014 were considered for the present study (Figure 1). We excluded 70 patients whose serum uric acid level at the time point immediately before treatment with xanthine oxidase inhibitors was $<7.0 \mathrm{mg} \mathrm{dl}^{-1}$. We also excluded 79 patients without hypertension. Hypertension was defined as more than two ambulatory blood pressure readings of $\geqslant 140 \mathrm{~mm} \mathrm{Hg}$ systolic and/or $90 \mathrm{~mm} \mathrm{Hg}$ diastolic or treatment with antihypertensive agents. We further

${ }^{1}$ Dialysis Unit, University Hospital of the Ryukyus, Okinawa, Japan; ${ }^{2}$ Department of Cardiovascular Medicine, Nephrology and Neurology University of the Ryukyus Graduate School of Medicine, Okinawa, Japan; ${ }^{3}$ Shuri-Jokamachi Clinic, Naha city, Okinawa, Japan; ${ }^{4}$ Clinical Research Center, Tomishiro Central Hospital, Tomigusuku city, Okinawa, Japan and ${ }^{5}$ Okinawa Heart and Renal Association, Naha city, Okinawa, Japan

Correspondence: Dr K Kohagura, Dialysis Unit, University Hospital of the Ryukyus, 207 Uehara, Nishihara-cho, Okinawa 903-0215, Japan.

E-mail: kohagura@med.u-ryukyu.ac.jp

Received 3 December 2015; revised 15 February 2016; accepted 29 February 2016; published online 14 April 2016 


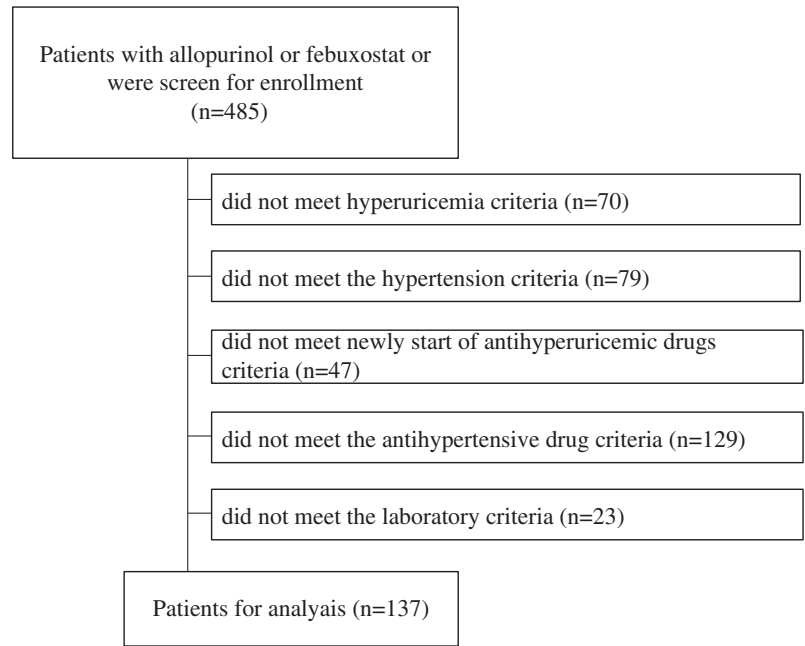

Figure 1 Flowchart of the study population.

excluded 47 patients who switched to allopurinol or febuxostat from other urate-lowering drugs. In addition, we excluded 129 patients who had any changes in medication, either before or after starting allopurinol or febuxostat treatment, and 23 patients who did not have data regarding serum uric acid, serum creatinine or blood pressure levels before, immediately before and after starting treatment with xanthine oxidase inhibitors. Finally, we recruited 137 hypertensive patients with hyperuricemia (uric acid $>7 \mathrm{mg} \mathrm{dl}^{-1}$ ) who had recently started allopurinol or febuxostat treatment. The study protocol was approved by the ethics review board of the University of the Ryukyus.

\section{Procedure}

Outpatient department nurses and physicians conducted a lifestyle and medical history questionnaire, collected blood and urine samples and measured the patients' blood pressure. Although the serum urate level is known to exhibit a circadian rhythm, because of the retrospective nature of this study, the timing of the urate measurements could not be determined. However, most of the patients appeared to visit the clinic for examination in the morning. Therefore, the timing of each patient visit was relatively consistent. Diabetes mellitus was defined as at least two measurements of a fasting plasma glucose level of $\geqslant 126 \mathrm{mg} \mathrm{dl}^{-1}$, at least two measurements of a 2-h plasma glucose level of $\geqslant 200 \mathrm{mg} \mathrm{dl}^{-1}$ or treatment with hypoglycemic agents. Dyslipidemia was defined as a low-density lipoprotein cholesterol level of $\geqslant 140 \mathrm{mg} \mathrm{dl}^{-1}$, a high-density lipoprotein cholesterol level of $<40 \mathrm{mg} \mathrm{dl}^{-1}$, a triglyceride level of $\geqslant 150 \mathrm{mg} \mathrm{dl}^{-1}$ or treatment with specific lipid-lowering agents, according to the criteria of the Japan Atherosclerosis Society. ${ }^{12}$ Height and weight were recorded, and the patients' body mass index was calculated. Serum creatinine were measured using the enzymatic method, and estimated glomerular filtration rate (eGFR) was calculated using the Japanese Society of Nephrology formula: ${ }^{13}$

eGFR $\left(\mathrm{ml} \mathrm{min}^{-1}\right.$ per $\left.1.73 \mathrm{~m}^{2}\right)=194 \times$ serum creatinine $e^{-1.094} \times$ age $^{-0.287} \times 0.739$ (if female).

\section{Outcomes}

The primary outcomes considered in the analysis were the changes in blood pressure and eGFR after treatment with xanthine oxidase inhibitors, defined as the differences between the values on the day of initiating urate-lowering drug treatment and the values at the next visit, without any changes in other medication during this period. We also examined blood pressure levels, uric acid levels and eGFRs during the visit immediately preceding treatment initiation with xanthine oxidase inhibitors to compare the changes in these parameters caused by treatment with xanthine oxidase inhibitors. For most patients, the time interval either before or after treatment was $\sim 1-3$ months.
Table 1 Basal characteristics at starting with xanthine oxidase inhibitors

\begin{tabular}{lc}
\hline & Median (interquartile range) or number (\%) \\
\hline Number & 137 \\
Age, years & $68(57-77)$ \\
Sex, male & $96(70)$ \\
Body mass index & $25.4(23.1-27.8)$ \\
Systolic blood pressure, mm Hg & $130(116-138)$ \\
Diastolic blood pressure, mm Hg & $70(62-80)$ \\
Creatinine, mg dl ${ }^{-1}$ & $1.3(1.0-1.5)$ \\
Estimated GFR, ml min ${ }^{-1}$ per $1.73 \mathrm{~m}^{2}$ & $43.2(31.2-59.0)$ \\
Uric acid, mg dl ${ }^{-1}$ & $8.9(8.3-9.4)$ \\
Diabetes mellitus & $42(31)$ \\
Dyslipidemia & $79(58)$ \\
Gout & $30(22)$ \\
Alcohol consumption & $61(52)$ \\
Diuretics & $50(36)$ \\
Thiazide & $31(23)$ \\
Loop diuretics & $23(17)$ \\
Calcium channel blocker & $95(69)$ \\
RAS inhibitors & $112(82)$ \\
$\beta$-Blocker & $45(33)$ \\
$\alpha-B l o c k e r$ & $10(7)$ \\
MR antagonist & $28(20)$ \\
Allopurinol/febuxostat & $72(53) / 65(47)$ \\
\hline
\end{tabular}

Abbreviations: GFR, glomerular filtration rate; MR, mineral corticoid receptor; RAS, renin-angiotensin system.

Data are expressed as median (interquartile range) or number (\%).

\section{Statistical analysis}

Differences in longitudinal parameters were tested by repeated measures analysis of variance. In the case of a $P$-value of ${ }^{<} 0.05$, a post hoc analysis was performed using Fisher's test. A paired $t$-test was used to analyze differences in percent (\%) changes in each parameter before and after treatment with xanthine oxidase inhibitors. Multiple regression analysis was performed to identify factors associated with an increased eGFR (that is, age; conventional risk factors for CKD, such as systolic blood pressure and diabetes mellitus (a potential factor known to be associated with glomerular filtration rate) and significant covariates in the nonadjusted model $(P<0.2))$ to avoid omitting potential covariates due to a suppressor effect. In addition, we included gender and eGFR (model 1) at the time point immediately preceding treatment initiation with xanthine oxidase inhibitors because of gender difference in the association between uric acid and $\mathrm{CKD}^{4,5} \mathrm{~A}$ strong association between uric acid and eGFR is known to exist. Moreover, we included the \% change in uric acid and \% change in systolic blood pressure after starting xanthine oxidase inhibitor treatment in model 2 to examine parameters associated with the change in the eGFR. Covariates were as follows: age (continuous variable); male gender ( $v$ s. female); factors such as systolic blood pressure, uric acid and eGFR at the time point immediately preceding treatment, $\%$ change in systolic blood pressure; \% change in uric acid (all continuous variables); gout (yes vs. no); diuretics use (yes vs. no); and renin-angiotensin system (RAS) inhibitor use (yes vs. no). The data are expressed as median (interquartile range), mean $(95 \%$ confidence intervals) or number (\%). $P$-values of $<0.05$ were considered statistically significant. The statistical analysis was conducted using StatView 5.0 statistical software (SAS Institute, Cary, NC, USA).

\section{RESULTS}

The baseline characteristics, that is, at the time point immediately before starting xanthine oxidase inhibitor administration, are summarized in Table 1. The study population was characterized as middle aged to aged, male dominant and obese. In addition, many patients had achieved good blood pressure control with either calcium channel 
blockers or RAS inhibitors and had CKD of stage 3 to 4 . Approximately $30 \%$ of the patients had diabetes mellitus or a history of gout or they used diuretics. The number of patients who were started on either drug was comparable (allopurinol, $N=72$; febuxostat, $N=65$ ). The doses of allopurinol were $50 \mathrm{mg}$ per day $(N=8), 100 \mathrm{mg}$ per day $(N=62)$ or $200 \mathrm{mg}$ per day $(N=2)$. The doses of febuxostat were $10 \mathrm{mg}$ per day $(N=53)$ or $20 \mathrm{mg}$ per day $(N=12)$.

Serial changes in uric acid level, eGFR and blood pressure before and after treatment with xanthine oxidase inhibitors

Xanthine oxidase inhibitors significantly reduced serum uric acid levels by $2.3 \mathrm{mg} \mathrm{dl}^{-1}$, although there was an increase that was accompanied by a reduction in blood pressure before starting treatment (Figure 2). A uric acid level of $<6 \mathrm{mg} \mathrm{dl}^{-1}$ was achieved in $29.9 \%$ of the total number of patients, in $32 \%$ of the patients taking febuxostat and in $27.8 \%$ of the patients taking allopurinol. In addition, eGFR decreased by $1.9 \mathrm{ml} \mathrm{min}^{-1}$ per $1.73 \mathrm{~m}^{2}$ before initiating xanthine oxidase inhibitors and conversely increased by $1.6 \mathrm{ml} \mathrm{min}^{-1}$ per $1.73 \mathrm{~m}^{2}$ after treatment. Although it was not statistically significant, the increase in the eGFR (\%) after treatment was greater in patients who achieved a uric acid level of $<6 \mathrm{mg} \mathrm{dl}^{-1}$ than in those who did not ( 6.9 vs. 3.1, $P=0.20)$. The increase in the eGFR (\%) was smaller in patients who were taking RAS inhibitors than in those who were not (3.2 vs. 8.9, $P=0.10)$, although, again, this increase was not statistically significant. Furthermore, the changes in uric acid (\%) were comparable between the patients who were taking RAS inhibitors and those who were not $(-25.0$ vs. $-25.4, P=0.91)$. Patients taking febuxostat had a greater improvement in eGFR (\%) than those taking allopurinol (6.7 vs. 2.0, $P=0.09$ ), although this improvement was not statistically significant. The urate-lowering effects were comparable between febuxostat and allopurinol $(-27.2$ vs. $-23.1, P=0.09)$. No significant changes in blood pressure were observed after starting xanthine oxidase inhibitors.

Comparison of the \% changes in uric acid level, eGFR and blood pressure before and after treatment with xanthine oxidase inhibitors

A clear contrast in the \% changes in the uric acid level and eGFR before and after treatment with xanthine oxidase inhibitors was observed (Table 2). After starting treatment, the \% change in the eGFR reversed, that is, became positive. There was no significant change in blood pressure before and after starting the treatment.
Uric acid

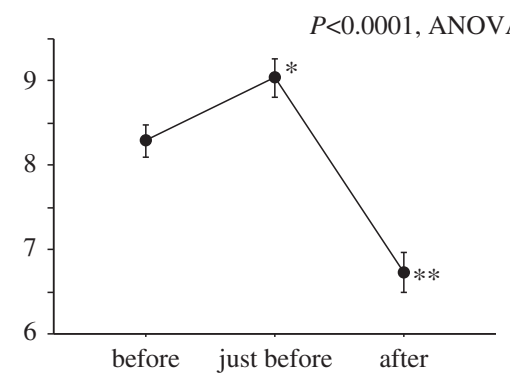

Systolic blood pressure

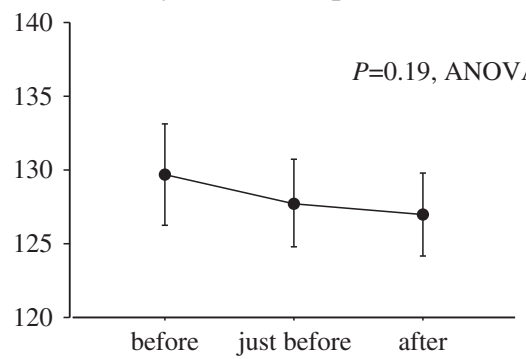

eGFR

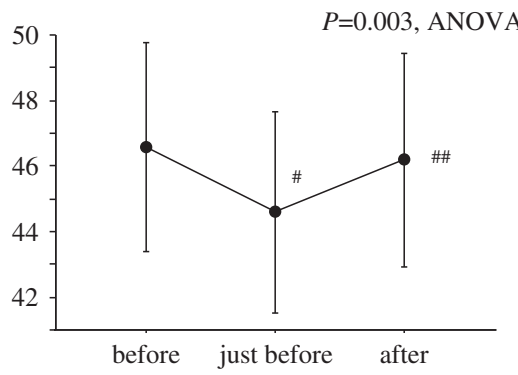

Diastolic blood pressure

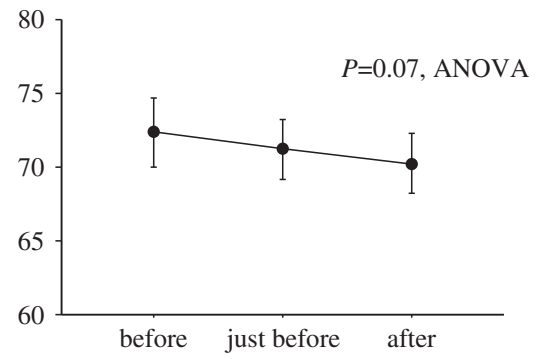

Figure 2 Serial changes in parameters before, immediately before and after starting xanthine oxidase inhibitor treatment. The circle markers represent the mean value, and the error bars represent the $95 \%$ confidence intervals for the mean. ${ }^{*} P<0.0001$ vs. before treatment, ${ }^{* *} P<0.0001$ vs. immediately before treatment; $\#=0.001$ vs. before, $\# \#=001$ vs. immediately before treatment. ANOVA, analysis of variance; eGFR, estimated glomerular filtration rate.

Table 2 Percent changes in parameters before and after treatment with xanthine oxidase inhibitors

\begin{tabular}{|c|c|c|c|}
\hline \multirow[b]{2}{*}{ Parameter } & \multicolumn{2}{|c|}{ Mean (5 to 95 percentile range) } & \multirow[b]{2}{*}{ P-value } \\
\hline & Before treatment & After treatment & \\
\hline Duration, day & 100 (28 to 287 ) & 65 (27 to 141$)$ & 0.003 \\
\hline$\%$ Change in uric acid, \% & $10.0(-12.0$ to 39.5$)$ & $-25.1(-45.9$ to -0.4$)$ & $<0.0001$ \\
\hline$\%$ Change in eGFR, \% & $-3.1(-23.8$ to 22.0$)$ & $4.2(-17.5$ to 32.4$)$ & 0.0009 \\
\hline$\%$ Change in SBP, \% & $-0.4(-21.1$ to 19.7$)$ & $0.09(-18.6$ to 16.9$)$ & 0.77 \\
\hline
\end{tabular}

Abbreviations: eGFR, estimated glomerular filtration rate; DBP, diastolic blood pressure; SBP, systolic blood pressure. 
Table 3 Factors associated with \% changes in eGFR after starting xanthine oxidase inhibitors

\begin{tabular}{|c|c|c|c|c|c|c|}
\hline \multirow[b]{3}{*}{ Variable } & & & \multicolumn{4}{|c|}{ Multivariate } \\
\hline & \multicolumn{2}{|c|}{ Unadjusted } & \multicolumn{2}{|c|}{$\begin{array}{c}\text { Model } 1 \\
\left(\mathrm{R}^{2}=0.17\right. \\
\mathrm{P}=0.04)\end{array}$} & \multicolumn{2}{|c|}{$\begin{array}{c}\text { Model } 2 \\
\left(\mathrm{R}^{2}=0.25\right. \\
\mathrm{P}=0.001)\end{array}$} \\
\hline & $\beta$ & P-value & $\beta$ & P-value & $\beta$ & P-value \\
\hline Age, years & -0.06 & 0.47 & -0.07 & 0.50 & -0.09 & 0.34 \\
\hline Sex, female & -0.04 & 0.66 & -0.02 & 0.86 & -0.08 & 0.4 \\
\hline Diabetes mellitus & 0.08 & 0.38 & 0.05 & 0.44 & 0.05 & 0.57 \\
\hline Gout & 0.14 & 0.15 & 0.04 & 0.73 & 0.06 & 0.96 \\
\hline $\begin{array}{l}\text { Renin-angiotensin } \\
\text { inhibitors }\end{array}$ & -0.14 & 0.10 & -0.27 & 0.006 & -0.27 & 0.004 \\
\hline Diuretics & -0.16 & 0.10 & -0.1 & 0.33 & -0.07 & 0.94 \\
\hline Febuxostat & 0.15 & 0.09 & 0.18 & 0.17 & 0.20 & 0.07 \\
\hline 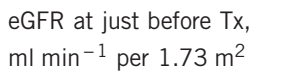 & -0.1 & 0.23 & -0.13 & 0.22 & -0.15 & 0.12 \\
\hline $\begin{array}{l}\text { Uric acid at just before } \mathrm{Tx} \\
\mathrm{mg} \mathrm{dl}^{-1}\end{array}$ & 0.1 & 0.23 & 0.07 & 0.45 & & \\
\hline $\begin{array}{l}\text { Systolic blood pressure at } \\
\text { just before } \mathrm{Tx}, \mathrm{mm} \mathrm{Hg}\end{array}$ & 0.05 & 0.53 & -0.04 & 0.69 & & \\
\hline $\begin{array}{l}\text { Change in uric acid, after } \\
\mathrm{Tx}, \%\end{array}$ & -0.23 & 0.008 & & & -0.24 & 0.01 \\
\hline $\begin{array}{l}\text { Change in systolic blood } \\
\text { pressure, after Tx, \% }\end{array}$ & 0.12 & 0.18 & & & 0.21 & 0.03 \\
\hline
\end{tabular}

Abbreviations: eGFR, estimated glomerular filtration rate; Tx, treatment.

Multivariate regression analysis including the following explanatory variables using significant factors in nonadjusted model $(P<0.2)$ in addition to gender and eGFR.

Model 1: gender, eGFR (continuous)+basal significant variable including systolic blood pressure (SBP) (continuous), basal uric acid(continuous) and diuretics.

Model 2: gender, eGFR (continuous)+significant \% change in variables including \% change in diastolic blood pressure (DBP) and \% change in uric acid+diuretics.

\section{Factors associated with \% change in eGFR}

We examined the factors associated with changes in eGFR after starting xanthine oxidase inhibitor treatment (Table 3). In the nonadjusted model, the $\%$ change in uric acid was negatively associated with the $\%$ change in the eGFR, whereas gout $(\beta=0.14, P=0.15)$, RAS inhibitor use $(\beta=-0.14, P=0.10)$, diuretic use $(\beta=-0.16, P=0.10)$ and febuxostat use $(\beta=0.15, P=0.09)$ were marginal factors. In the multivariate regression analysis of model 1 , which included age, gender, diabetes mellitus and factors such as eGFR, uric acid, systolic blood pressure immediately before treatment and marginal factors, RAS inhibitor use negatively correlated with the $\%$ change in the eGFR. In the multivariate regression analysis of model 2 in which the \% change in uric acid and systolic blood pressure after starting treatment with xanthine oxidase inhibitors were substituted for uric acid level and systolic blood pressure at the time point immediately before treatment, the $\%$ change in the uric acid level and RAS inhibitor use negatively correlated with the increase in the eGFR, whereas the \% change in the systolic blood pressure positively correlated with the increase in the eGFR.

\section{DISCUSSION}

Previous interventional studies suggested that treatment with allopurinol may have a renoprotective effect in CKD patients. ${ }^{6,14}$ Similarly, in the present study, treatment with xanthine oxidase inhibitors for a few months was associated with a mild elevation of the eGFR, without any significant effect on blood pressure in hypertensive patients with hyperuricemia.
In the present study, the duration of treatment with xanthine oxidase inhibitors was relatively short and may be insufficient to determine whether the changes in renal function were derived from these drugs. However, treatment with xanthine oxidase inhibitors led to a reversal of the eGFR decline that was observed before starting treatment with these drugs. Therefore, the change in the eGFR seemed to be due to the treatment with xanthine oxidase inhibitors. Although a previous randomized, controlled study conducted among adolescents had shown that treatment with allopurinol for 4 to 8 weeks significantly decreased blood pressure, ${ }^{7,15}$ we found no significant decrease in blood pressure among adult hypertensive patients. Basal blood pressure was relatively well controlled with antihypertensive medication. The methods for assessing blood pressure in the clinic might not be sensitive enough to detect relatively small differences in blood pressure. Alternatively, it was proposed that uric acid might be associated with the early phase of hypertension development, whereas salt-sensitive hypertension rather than uric acid itself contributes to the maintenance of high blood pressure in the late phase. ${ }^{16}$ The reduced renal function and higher rate of comorbidity in the present study may therefore influence the effects of xanthine oxidase inhibitors on blood pressure.

Although the mechanisms involved in the effects of xanthine oxidase inhibitors on eGFR are not fully known, some mechanisms might be surmised. Because the elevation of systolic blood pressure after treatment was associated with an increase in the eGFR, the observed improvement in renal function may be partially due to changes in systemic hemodynamics. Before the administration of xanthine oxidase inhibitors, serum uric acid levels increased as the blood pressure and the eGFR decreased. Because these clinical observations suggested that hemodynamic changes accompanied by decreases in blood pressure determined by specific conditions, such as dehydration, cause a decrease in the eGFR and an increase in the uric acid levels, patients might be advised to increase their water intake and/or lighten their sodium restriction. These measures might result in the recovery of the eGFR and blood pressure in some patients. The negative association between the increase in the eGFR and the use of RAS inhibitors also supported the involvement of hemodynamic changes. Aside from the change in blood pressure, a significant decrease in uric acid levels was also associated with an increase in the eGFR, suggesting that a urate-lowering effect could be involved in the mechanisms of eGFR increase. We reported that higher levels of uric acid were associated with renal arteriolopathy among CKD patients who underwent renal biopsy, ${ }^{17}$ as previously shown in an animal model. ${ }^{18}$ In addition, an earlier study demonstrated that renal vascular resistance was positively correlated with serum uric acid levels among patients with essential hypertension, ${ }^{19}$ and short-term treatment with allopurinol decreased peripheral vascular resistance. ${ }^{15}$ Because renal arterioles have a pivotal role in the regulation of glomerular hemodynamics, enhancement in renal microcirculation may be responsible for eGFR improvement associated with the urate-lowering effect.

The findings of the present study are clinically significant in terms of renal protection for hypertensive patients with hyperuricemia. Increased serum uric acid levels have been shown to be associated with an increase in the prevalence of hypertension. ${ }^{20}$ Moreover, a previous epidemiological study demonstrated that the association between hyperuricemia and the risk of developing renal dysfunction was more prevalent in patients with hypertension than in normotensive subjects. ${ }^{21}$ In many patients with $\mathrm{CKD}$, adequate volume control with diuretics would be essential to achieve target blood pressure control..$^{22,23}$ The use of diuretics is frequently associated with hyperuricemia. ${ }^{24}$ It has been suggested that newly developed hyperuricemia may be associated with an earlier decline in eGFR and increased risk of cardiovascular events in clinical studies conducted 
among patients with hypertension that included many users of diuretics. ${ }^{9,25}$ In such clinical settings, improvement in the eGFR by xanthine oxidase inhibitors may delay the progression of CKD. Moreover, febuxostat, which is a newly developed xanthine oxidase inhibitor, was shown to be safe in patients with CKD at stages $3 \mathrm{~b}$ to $5{ }^{26}$ Therefore, xanthine oxidase inhibitors have potential renoprotective benefits even in patients with advanced stages of CKD.

The present study has some limitations. First, because of the nature of retrospective observational studies, we cannot determine whether the increase in the eGFR could be solely attributed to the effects of xanthine oxidase inhibitors. However, although it was not statistically significant, febuxostat produced a greater improvement in eGFR (\%) than allopurinol. Because the urate-lowering effects of febuxostat and allopurinol are comparable, xanthine oxidase inhibition and not urate reduction may contribute more to the eGFR. Prospective, randomized, controlled studies to elucidate the effects of xanthine oxidase inhibitors on renal function and blood pressure in adult patients with established hypertension are clearly indicated. Second, it is not clear whether the effects of short-term treatment with xanthine oxidase inhibitors on the eGFR would translate into a long-term renal outcome. Recently, an extension study of a randomized controlled trial showed that improving the eGFR by treatment with allopurinol for 1 year was associated with a favorable long-term renal outcome among CKD patients. ${ }^{27}$ Similarly, we need to determine the long-term clinical relevance of xanthine oxidase inhibitors by conducting a prospective, randomized, controlled trial in hypertensive patients with hyperuricemia. In particular, we intend to assess proteinuria among patients with increased eGFRs. Unfortunately, the available data regarding proteinuria were limited in the present study. Third, blood pressure measurements obtained in the clinic might not be adequate to detect relatively small changes in blood pressure in patients with hypertension that is well controlled using medication. Thus, we need more accurate methods, such as ambulatory blood pressure monitoring, to detect an effect on blood pressure. Fourth, most of the patients in the study had stage 3 to $4 \mathrm{CKD}$ that corresponds to a significant degree of renal dysfunction. We therefore cannot extrapolate the results of the present study to all hypertensive patients with hyperuricemia, especially those with normal kidney function. Serum uric acid levels increased just before starting xanthine oxidase inhibitor treatment, suggesting a short duration of hyperuricemia. The results of the present study can therefore be applied to a limited group of hypertensive patient with hyperuricemia.

In conclusion, treatment with xanthine oxidase inhibitors may improve the eGFR among adult hypertensive patients with hyperuricemia. This additional effect may improve renal outcome, if it is maintained long term.

\section{CONFLICT OF INTEREST}

The authors declare no conflict of interest.

\section{ACKNOWLEDGEMENTS}

We thank M Kobayashi and M Arakaki for data collection, cleaning and secretarial support.

1 Grayson PC, Kim SY, LaValley M, Choi HK. Hyperuricemia and incident hypertension: a systematic review and meta-analysis. Arthritis Care Res (Hoboken) 2011; 63: 102-110.

2 Nagahama K, Inoue T, Iseki K, Touma T, Kinjo K, Ohya Y, Takishita S. Hyperuricemia as a predictor of hypertension in a screened cohort in Okinawa, Japan. Hypertens Res 2004; 27: 835-841.

3 Nagahama $\mathrm{K}$, Inoue $\mathrm{T}$, Kohagura $\mathrm{K}$, Kinjo $\mathrm{K}$, Ohya $\mathrm{Y}$. Associations between serum uric acid levels and the incidence of hypertension and metabolic syndrome: a 4-year follow-up study of a large screened cohort in Okinawa, Japan. Hypertens Res 2015; 38: 213-218.
4 Iseki K, Oshiro S, Tozawa M, Iseki C, Ikemiya Y, Takishita S. Significance of hyperuricemia on the early detection of renal failure in a cohort of screened subjects. Hypertens Res 2001: 24: 691-697.

5 Iseki K, Ikemiya Y, Inoue T, Iseki C, Kinjo K, Takishita S. Significance of hyperuricemia as a risk factor for developing ESRD in a screened cohort. Am J Kidney Dis 2004; 44: 642-650.

6 Johnson RJ, Nakagawa T, Jalal D, Sánchez-Lozada LG, Kang DH, Ritz E. Uric acid and chronic kidney disease: which is chasing which? Nephrol Dial Transplant 2013; 28: 2221-2228.

7 Feig DI, Soletsky B, Johnson RJ. Effect of allopurinol on blood pressure of adolescents with newly diagnosed essential hypertension: a randomized trial. JAMA 2008; 300: 924-932.

8 Nagahama K, Iseki K, Inoue T, Touma T, Ikemiya Y, Takishita S. Hyperuricemia and cardiovascular risk factor clustering in a screened cohort in Okinawa, Japan. Hypertens Res 2004; 27: 227-233.

9 Miao Y, Ottenbros SA, Laverman GD, Brenner BM, Cooper ME, Parving HH, Grobbee DE, Shahinfar S, de Zeeuw D, Lambers Heerspink HJ. Effect of a reduction in uric acid on renal outcomes during losartan treatment: a post hoc analysis of the reduction of endpoints in non-insulin-dependent diabetes mellitus with the Angiotensin II Antagonist Losartan Trial. Hypertension 2011; 58: 2-7.

10 Goicoechea M, de Vinuesa SG, Verdalles U, Ruiz-Caro C, Ampuero J, Rincón A, Arroyo D, Luño J. Effect of allopurinol in chronic kidney disease progression and cardiovascular risk. Clin J Am Soc Nephrol 2010; 5: 1388-1393.

11 Siu YP, Leung KT, Tong MK, Kwan TH. Use of allopurinol in slowing the progression of renal disease through its ability to lower serum uric acid level. Am J Kidney Dis 2006; 47: 51-59.

12 Teramoto T, Sasaki J, Ueshima H, Egusa G, Kinoshita M, Shimamoto K, Daida H, Biro S, Hirobe K, Funahashi T, Yokote K, Yokode MJapan Atherosclerosis Society (JAS) Committee for Epidemiology and Clinical Management of Atherosclerosis. Diagnostic criteria for dyslipidemia. Executive summary of Japan Atherosclerosis Society (JAS) guideline for diagnosis and prevention of atherosclerotic cardiovascular diseases for Japanese. J Atheroscler Thromb 2007; 14: 155-158.

13 Matsuo S, Imai E, Horio M, Yasuda Y, Tomita K, Nitta K, Yamagata K, Tomino Y, Yokoyama $\mathrm{H}$, Hishida ACollaborators developing the Japanese equation for estimated GFR. Revised equations for estimated GFR from serum creatinine in Japan. Am J Kidney Dis. 2009; 53: 982-992.

14 Bose B, Badve SV, Hiremath SS, Boudville N, Brown FG, Cass A, de Zoysa JR, Fassett RG, Faull R, Harris DC, Hawley CM, Kanellis J, Palmer SC, Perkovic V, Pascoe EM, Rangan GK, Walker RJ, Walters G, Johnson DW. Effects of uric acid-lowering therapy on renal outcomes: a systematic review and meta-analysis. Nephrol Dial Transplant 2014; 29: 406-413.

15 Soletsky B, Feig DI. Uric acid reduction rectifies prehypertension in obese adolescents. Hypertension 2012; 60: 1148-1156.

16 Feig DI, Kang DH, Johnson RJ. Uric acid and cardiovascular risk. N Engl J Med 2008; 359: 1811-1821.

17 Kohagura K, Kochi M, Miyagi T, Kinjyo T, Maehara Y, Nagahama K, Sakima A, Iseki K, Ohya $Y$. An association between uric acid levels and renal arteriolopathy in chronic kidney disease: a biopsy-based study. Hypertens Res 2013; 36: 43-49.

18 Mazzali M, Kanellis J, Han L, Feng L, Xia YY, Chen Q, Kang DH, Gordon KL, Watanabe S, Nakagawa T, Lan HY, Johnson RJ. Hyperuricemia induces a primary renal arteriolopathy in rats by a blood pressure-independent mechanism. Am J Physiol Renal Physiol 2002; 282: F991-F997.

19 Messerli FH, Frohlich ED, Dreslinski GR, Suarez DH, Aristimuno GG. Serum uric acid in essential hypertension: an indicator of renal vascular involvement. Ann Intern Med 1980; 93: 817-821.

20 Kuwabara M, Niwa K, Nishi Y, Mizuno A, Asano T, Masuda K, Komatsu I, Yamazoe M, Takahashi $\mathrm{O}$, Hisatome I. Relationship between serum uric acid levels and hypertension among Japanese individuals not treated for hyperuricemia and hypertension. Hypertens Res 2014; 37: 785-789.

21 Obermayr RP, Temml C, Gutjahr G, Knechtelsdorfer M, Oberbauer R, Klauser-Braun R. Elevated uric acid increases the risk for kidney disease. J Am Soc Nephrol 2008; 19. 2407-2413.

22 De Nicola L, Minutolo R, Bellizzi V, Zoccali C, Cianciaruso B, Andreucci VE, Fuiano G, Conte Glnvestigators of TArget Blood Pressure LEvels in Chronic Kidney Disease (TABLE in CKD) Study Group. Achievement of target blood pressure levels in chronic kidney disease: a salty question? Am J Kidney Dis 2004; 43: 782-795.

23 Agarwal R, Sinha AD, Pappas MK, Ammous F. Chlorthalidone for poorly controlled hypertension in chronic kidney disease: an interventional pilot study. Am J Nephrol 2014; 39: 171-182.

24 Lin CS, Lee WL, Hung YJ, Lee DY, Chen KF, Chi WC, Chang SC. Prevalence of hyperuricemia and its association with antihypertensive treatment in hypertensive patients in Taiwan. Int J Cardiol 2012; 156: 41-46.

25 Høieggen A, Alderman MH, Kjeldsen SE, Julius S, Devereux RB, De Faire U, Fyhrquist F, Ibsen $\mathrm{H}$, Kristianson K, Lederballe-Pedersen O, Lindholm LH, Nieminen MS, Omvik P, Oparil S, Wedel H, Chen C, Dahlöf BLIFE Study Group. The impact of serum uric acid on cardiovascular outcomes in the LIFE study. Kidney Int 2004; 65: 1041-1049.

26 Shibagaki Y, Ohno I, Hosoya T, Kimura K. Safety, efficacy and renal effect of febuxostat in patients with moderate-to-severe kidney dysfunction. Hypertens Res 2014; 37: 919-925.

27 Goicoechea M, Garcia de Vinuesa S, Verdalles U, Verde E, Macias N, Santos A, Pérez de Jose $A$, Cedeño $S$, Linares $T$, Luño J. Allopurinol and progression of $C K D$ and cardiovascular events: long-term follow-up of a randomized clinical trial. Am J Kidney Dis 2015; 65: 543-549. 